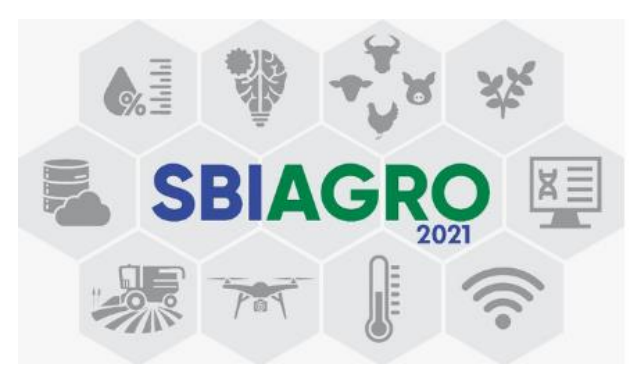

\title{
Diferenciação e caracterização de municípios produtores de bovinos de corte no bioma Mata Atlântica segundo o Censo Agropecuário 2017
}

\author{
Maria do Carmo Ramos Fasiaben', Stanley Robson de Medeiros Oliveira ${ }^{1}$, André \\ Steffens Moraes ${ }^{2}$, Maxwell Merçon Tezolin Barros Almeida ${ }^{3}$, Octávio Costa de \\ Oliveira $^{3}$, Gabriela dos Santos Eusébio ${ }^{4}$ \\ ${ }^{1}$ Embrapa Agricultura Digital, Campinas, SP. \\ ${ }^{2}$ Emprapa Soja, Londrina, PR. \\ ${ }^{3}$ IBGE, COAGRO, Rio de Janeiro, RJ. \\ ${ }^{4}$ Instituto de Economia/Unicamp, Campinas, SP \\ \{maria.fasiaben; stanley.oliveira; andre.moraes\}@embrapa.br, \\ \{maxwell.almeida; octavio.oliveira\}@ibge.gov.br, gabeusebio@gmail.com
}

\begin{abstract}
The objective of this work is to typify beef cattle producing municipalities in the Mata Atlântica biome based on data from the 2017 Agricultural Census. Special census tabulations were needed to aggregate agricultural establishments by municipality, with emphasis on the technical characteristics of livestock production systems. Four groups of municipalities were identified according to the biome's production patterns: two high-tech groups, one of them with emphasis on confinement; an intermediate technology group and a low technology group. The mapping of the different production patterns throughout the territory can support research, technology transfer and development actions that are more appropriate to the different situations of producers.
\end{abstract}

Resumo. $O$ objetivo deste trabalho é tipificar os municipios produtores de bovinos de corte do bioma Mata Atlântica a partir de dados do Censo Agropecuário 2017. Tabulações especiais do censo foram necessárias para agregar os estabelecimentos agropecuários por município, com ênfase nas características técnicas dos sistemas de produção pecuária. Identificaram-se quatro grupos de municípios segundo os padrões de produção do bioma: dois grupos de alta tecnologia, um deles com destaque para confinamento; um grupo de tecnologia intermediária e outro de baixa tecnologia. O mapeamento dos distintos padrões de produção ao longo do território pode apoiar ações de pesquisa, de transferência de tecnologia e de desenvolvimento mais apropriadas às diferentes situações dos produtores. 


\section{Introdução}

O bioma Mata Atlântica se estende de norte a sul do país desde o Estado do Rio Grande do Sul até o Piauí ao longo da costa brasileira, e se expande para o oeste, na região Sudeste, até as fronteiras com a Argentina e o Paraguai. Aí vive cerca de $70 \%$ da população brasileira e se desenvolve $80 \%$ da atividade econômica do país [FUNDAÇÃO SOS MATA ATLÂNTICA, 2021].

Ocupando originalmente $15 \%$ do território nacional, atualmente o remanescente de vegetação nativa acima de três hectares deste bioma é de $12,4 \%$ em relação ao original, resultado de um intenso processo de exploração [FUNDAÇÃO SOS MATA ATLÂNTICA; INPE, 2021].

Embora em alguns dos 17 estados com área no bioma o desmatamento esteja se aproximando de zero (tecnicamente, quando a área desmatada é menor que 100 ha no período avaliado), a tendência em 10 dos estados é de perda acentuada da vegetação nativa. Os fragmentos maiores do que 100 ha representam somente 8,5\% da área de floresta original do bioma [PINTO et al. 2021].

Apesar desse alto grau de devastação, a Mata Atlântica é um dos biomas terrestres mais ricos em espécies animais e vegetais do planeta, sendo considerado pela Unesco um dos hotspots mundiais em biodiversidade e um dos biomas brasileiros prioritários para conservação [UNESCO, 2021]. Sua rica variedade biológica se deve aos diferentes ecossistemas existentes no bioma e suas características climáticas e geológicas [INCT-BioNat, s.d; EMBRAPA, 2018]. Por sua extensão ao longo do território nacional, esse bioma ocupa condições diversas de solo, relevo e clima, abrigando ampla variedade de cultivos: grãos, algodão, café, cana-de- açúcar, silvicultura, fruticultura e outros. As pecuárias de leite e de corte podem ser encontradas praticamente em toda sua extensão [FASIABEN et al., 2018].

Apesar de o Brasil ser o segundo maior produtor e o maior exportador mundial de carne bovina, a principal característica no desenvolvimento dessa atividade no país é a heterogeneidade nos sistemas de produção e nos mecanismos de gestão e de comercialização do gado. Sistemas de produção baseados na produção extensiva, pequena intensidade tecnológica e padrões precários de gestão e de comercialização trazem ao setor um forte impacto ambiental [CARVALHO \& ZEN, 2017].

Além do aumento da produção para suprir uma demanda crescente, a bovinocultura de corte brasileira terá que se adaptar ao novo cenário nacional e internacional que demanda produtos oriundos de sistemas sustentáveis, ademais da concorrência por área com outras atividades agrícolas [Diagnóstico..., 2016]. O aumento da produtividade dentro de padrões sustentáveis é um imperativo.

No bioma Mata Atlântica, a produção de bovinos cuja principal finalidade é corte representa uma parcela importante da produção nacional. Os dados do Censo Agropecuário 2017 mostram que, se considerada a totalidade dos estabelecimentos agropecuários produtores de bovinos de corte do país (aqueles com uma ou mais cabeças com essa finalidade), a Mata Atlântica detém perto de um terço dos estabelecimentos produtores, $20 \%$ do rebanho e $16 \%$ da área de pastagens. Tendo-se em conta os estabelecimentos produtores com mais de 50 cabeças de bovinos de corte, de maior expressão comercial, nesse bioma se encontram cerca de $26 \%$ dos estabelecimentos, $18 \%$ do rebanho e $14 \%$ da área de pastagens destinadas a essa finalidade no país, 
ficando, nesse último caso, atrás somente do Cerrado e da Amazônia nesses quesitos (IBGE, 2020).

Fatores como a extensão territorial do bioma, sua diferenciação edafoclimática e a diversidade socioeconômica dos produtores implicam em uma grande variedade de sistemas de produção na pecuária de corte (FASIABEN et al., 2018). Sistemas de produção com maior variedade funcional, incluindo sistemas de produção pecuários, tendem a ser mais sustentáveis (MARCONDELLI et al., 2018), o que é bastante positivo em um bioma que requer atenção para a conservação de seus recursos naturais.

Utilizando dados oriundos do Censo Agropecuário 2017 do IBGE, o objetivo deste trabalho foi elaborar uma tipificação dos municípios do bioma Mata Atlântica segundo características da criaçao de bovinos de corte, com enfoque especial na tecnologia empregada pelos produtores. Espera-se que os resultados sejam úteis para o planejamento de ações de pesquisa agropecuária, transferência de tecnologia e desenvolvimento rural, colaborando para a conservação do bioma.

\section{Material e métodos}

Foi obtida uma tabulação especial do Censo Agropecuário 2017 com variáveis agrupadas por municípios e filtrada por estabelecimentos agropecuários com mais de 50 cabeças de bovinos de corte (IBGE, 2020). Variáveis quantitativas (exceto número de estabelecimentos) com menos de três informantes por município foram desidentificadas, por motivo de sigilo estatístico. As variáveis para a tipificação são listadas a seguir:

1. Número de cabeças de bovinos de corte

2. Taxa de lotação (cabeças/ha de pastagem)

3. Área de pastagem/área em processo produtivo

4. Área de lavoura/área em processo produtivo

5. Área de silvicultura/área em processo produtivo

6. Área de Sistemas Agroflorestais/área em processo produtivo

7. Número de fêmeas de mais de 2 anos/número total de cabeças de bovinos de corte

8. Valor da produção advindo de bovinos de corte/valor da produção agropecuária

9. Receita agropecuária/receita familiar

10. Porcentagem de estabelecimentos que fazem suplementação alimentar

11. Porcentagem de estabelecimentos que fazem confinamento

12. Porcentagem de estabelecimentos que recebem orientação técnica

13. Porcentagem de estabelecimentos que usam adubos químicos

14. Porcentagem de estabelecimentos que usam corretivos do solo

15. Porcentagem de estabelecimentos que têm tratores

16. Porcentagem de estabelecimentos familiares

17. Valor da venda de bovinos de corte para abate/valor de venda de bovinos

Na etapa de modelagem, adotou-se o algoritmo Expectation-Maximization (EM) para se estimar o número de grupos (clusters) [DEMPSTER et al., 1977]. A razão da escolha desse algoritmo baseia-se nos critérios: a) EM estende o paradigma usado no kmeans, associando uma distribuição de probabilidade a cada instância, que indica a probabilidade dessa instância pertencer a uma única população; b) EM tem a habilidade de lidar com valores faltantes e de estimar o número de clusters por meio de um 
conjunto de parâmetros que descreve a distribuição de probabilidade de cada cluster. $\mathrm{O}$ usuário também pode especificar a priori quantos clusters gerar.

O algoritmo calcula os estimadores de máxima verossimilhança para problemas onde existem dados incompletos entre os dados observados ou quando os estados das variáveis não foram observados, dados não observados. Cada iteração do algoritmo EM envolve dois passos que são: expectation (passo E) e maximization (passo M). O Passo E desse algoritmo consiste em obter uma estimativa da esperança (expectation) dos dados faltosos para completar a amostra de dados incompleta, partindo-se de um valor inicial empírico. No Passo M, com os dados completados, realiza-se aprendizagem das probabilidades, baseado nas frequências dos estados das variáveis na amostra, de modo a calcular a função de maximização da esperança encontrada. Os Passos E e M fazem parte de um processo iterativo, em que as novas probabilidades, calculadas na fase $\mathrm{M}$, serão utilizadas para realizar a inferência na fase E.

A validação cruzada realizada para determinar o número de clusters é feita nas seguintes etapas: a) o número de clusters é definido como 1 ; b) o conjunto de treinamento é dividido aleatoriamente em 10 partições (folds); c) EM é processado 10 vezes usando as 10 partições da maneira usual de validação cruzada; d) a probabilidade da máxima verossimilhança é calculada e a média dos 10 resultados é obtida; e) se a probabilidade de máxima verossimilhança aumentar, o número de clusters é aumentado em 1 e o programa continua na etapa 2 . O número de partições é fixada em 10 , desde que o número de instâncias (observações) no conjunto de treinamento não seja menor que 10. Se for esse o caso, o número de partições é definido igual ao número de instâncias.

Em particular, foi utilizada a versão do algoritmo EM disponível no software Weka, versão 3.8.5 [FRANK et al., 2006]. Weka é uma coleção de algoritmos de aprendizado de máquina para tarefas de mineração de dados. Ele contém ferramentas para preparação de dados, classificação, regressão, clustering, mineração de regras de associação e visualização.

Uma vez configurados os agrupamentos a partir do conjunto de variáveis, outras informações foram empregadas para melhor caracterizá-los, conforme se descreve nos resultados a seguir.

\section{Resultados}

O Bioma Mata Atlântica apresentou 91.554 estabelecimentos agropecuários de bovinos de corte com mais de 50 cabeças, detendo um total de 22,1 milhões de cabeças e 15,6 milhões de hectares de áreas de pastagens. Esses estabelecimentos estavam presentes em 2.405 municípios, que foram classificados em quatro grupos (clusters) segundo a metodologia estatística descrita (Tabela 1).

Em primeiro lugar, observa-se a presença de áreas de lavouras que chegam a ser expressivas nos diferentes clusters, denotando a diversificação como característica geral dos sistemas de produção de bovinos de corte praticados no bioma Mata Atlântica.

Os Clusters com maior nível tecnológico (Clusters 2 e 3) são os menos frequentes. Se somados, representam $12 \%$ dos estabelecimentos, que se distribuem em $28 \%$ dos municípios. Entretanto, esses dois clusters detêm somente 2\% e 3\% do efetivo de bovinos de corte e $1 \%$ e $4 \%$ da área de pastagem do bioma, respectivamente (Tabela 
1). O Cluster 2 é o que apresenta maior taxa de lotação (média de 2,45 cabeças/ha), seguido do Cluster 3 (2,3 cabeças/ha). Ambos se diferenciam, principalmente, pela maior presença de estabelecimentos que fazem confinamento no Cluster 2 . O Cluster $2 \mathrm{e}$ o Cluster 3 são também os que apresentam maior diversificação: a proporção de área destinadas a lavouras (em relação às áreas em processo produtivo) é, respectivamente, de $33 \%$ e $56 \%$. O maior percentual de ocupação com florestas plantadas se observa no Cluster 2, sendo aí muito superior à média do bioma (Tabela 2). Observando as variáveis que refletem o nível tecnológico (Tabela 3), vemos que esses dois clusters se apresentam bem acima da média da Mata Atlântica em relação aos quesitos confinamento, suplementação alimentar, uso de corretivos do solo, presença de tratores, orientação técnica e acesso à Internet. Foge da regra o fato de o Cluster 2 ter apresentado menor média de emprego de adubo químico em relação à média do bioma. Já no caso do Cluster 3 o uso de adubação química é bem superior à média geral do bioma. A maior presença de lavouras poderia explicar o maior uso de adubos, uma vez que o censo de 2017 não coleta a informação do destino dos insumos. Uma melhor localização em termos de solos e a possibilidade de rotações entre lavouras e pastagens também podem ser fatores a explicar as maiores taxas de lotação nos Clusters 2 e 3.

O Cluster 1 se apresenta como o agrupamento de municípios que agrega estabelecimentos com nível intermediário de tecnologia, quando se considera o bioma Mata Atlântica. Ele representa 34\% dos estabelecimentos e 31\% dos municípios analisados. Esse cluster detém $30 \%$ do efetivo de bovinos de corte e $26 \%$ da área de pastagens destinada a esse tipo de rebanho do bioma (Tabela 1). Das terras em processo produtivo nos estabelecimentos do Cluster $1,72 \%$ são ocupadas com pastagens, $21 \%$ com lavouras, $3 \%$ com florestas plantadas e $4 \%$ com sistemas agroflorestais. Nesse último caso, essa é a maior média entre todos os clusters (Tabela 2). A média da taxa de lotação desse cluster é de 1,64 cabeças/ha. O conjunto de dados da Tabela 3 mostra que esse cluster apresenta valores intermediários nas diferentes variáveis tecnológicas, com exceção do uso de adubos químicos e corretivos, conforme mencionado anteriormente.

O Cluster 4 é o que predomina em termos de estabelecimentos de bovinos de corte (62\% do total do bioma) e municípios ( $41 \%$ do total) e é o que se apresenta com menor nível tecnológico no bioma. Ele concentra $62 \%$ das cabeças e $69 \%$ da área de pastagens destinadas a essa finalidade na Mata Atlântica (Tabela 1). O uso das terras em processo produtivo aponta para $92 \%$ com pastagens (superior à média do bioma), $5 \%$ de áreas de lavouras (inferior à média do bioma) e $1 \%$ tanto para as áreas de florestas plantadas quanto para sistemas agroflorestais (também inferiores à média). Na Tabela 3 pode-se verificar que os valores de todas as variáveis indicativas do nível tecnológico apresentam-se abaixo da média do bioma. Chama a atenção a porcentagem de estabelecimentos agropecuários que recebe algum tipo de orientação técnica neste agrupamento, que é de apenas 40\%. A Internet chega a somente $39 \%$ dos estabelecimentos agropecuários desse grupo. 
Tabela 1. Ocorrências dos clusters de bovinos de corte em estabelecimentos com mais de 50 cabeças no Bioma Mata Atlântica, agregados por municípios, segundo variáveis selecionadas

\begin{tabular}{|c|c|c|c|c|c|}
\hline Variável / Cluster & Cluster 1 & Cluster 2 & Cluster 3 & Cluster 4 & M. Atlântica \\
\hline Número de ocorrências (municípios) & 750 & 311 & 356 & 988 & 2.405 \\
\hline Porcentagem de ocorrências em relação ao total do Bioma & $31 \%$ & $13 \%$ & $15 \%$ & $41 \%$ & $100 \%$ \\
\hline Número de estabelecimentos produtores de bovinos de corte com mais de 50 cabeças (unid) & 31.402 & 3.154 & 7.813 & 49.185 & 91.554 \\
\hline Porcentagem de estabelecimentos em relação ao total do Bioma & $34 \%$ & $3 \%$ & $9 \%$ & $54 \%$ & $100 \%$ \\
\hline Efetivo de bovinos de corte no cluster (cabeças) & 6.593 .997 & 418.293 & 1.428 .583 & 13.631 .252 & 22.072 .125 \\
\hline Porcentagem de cabeças do cluster em relação ao total do Bioma & $30 \%$ & $2 \%$ & $6 \%$ & $62 \%$ & $100 \%$ \\
\hline Área de pastagem do cluster (ha) & 4.027 .140 & 170.717 & 621.049 & 10.742 .422 & 15.561 .328 \\
\hline Porcentagem da área de pastagem em relação ao total do Bioma & $26 \%$ & $1 \%$ & $4 \%$ & $69 \%$ & $100 \%$ \\
\hline
\end{tabular}

Tabela 2. Características quanto ao uso da terra de estabelecimentos produtores de bovinos de corte com mais de $\mathbf{5 0}$ cabeças no Bioma Mata Atlântica, agregados por municípios, segundo clusters e total Mata Atlântica

\begin{tabular}{|c|c|c|c|c|c|}
\hline Variável / Cluster & Cluster 1 & Cluster 2 & Cluster 3 & Cluster 4 & M. Atlântica \\
\hline Área total (média) dos estabelecimentos agropecuários (ha) & 229 & 142 & 257 & 290 & 261 \\
\hline Área média em processo produtivo dos estabelecimentos (ha) & 179 & 103 & 205 & 237 & 210 \\
\hline Área média de pastagem dos estabelecimentos (ha) & 128 & 54 & 79 & 218 & 170 \\
\hline Porcentagem da área ocupada com pastagens nos estabelecimentos & $72 \%$ & $53 \%$ & $39 \%$ & $92 \%$ & $81 \%$ \\
\hline Porcentagem da área ocupada com lavouras nos estabelecimentos & $21 \%$ & $33 \%$ & $56 \%$ & $5 \%$ & $15 \%$ \\
\hline Porcentagem da área ocupada com florestas plantadas nos estabelecimentos & $3 \%$ & $10 \%$ & $2 \%$ & $1 \%$ & $2 \%$ \\
\hline Porcentagem da área ocupada com sistemas agroflorestais nos estabelecimentos & $4 \%$ & $1 \%$ & $1 \%$ & $1 \%$ & $2 \%$ \\
\hline
\end{tabular}

Tabela 3. Aspectos gerais da tecnologia empregada nos estabelecimentos produtores de bovinos de corte com mais de 50 cabeças no Bioma Mata Atlântica, agregados por municípios, segundo clusters e total Mata Atlântica

\begin{tabular}{|c|c|c|c|c|c|}
\hline Variável / Cluster & Cluster 1 & Cluster 2 & Cluster 3 & Cluster 4 & M. Atlântica \\
\hline Tamanho médio do rebanho por estabelecimento (cabeças) & 210 & 133 & 183 & 277 & 241 \\
\hline Porcentagem de vacas com 2 anos ou mais em relação ao rebanho total & $34 \%$ & $27 \%$ & $31 \%$ & $28 \%$ & $30 \%$ \\
\hline Taxa de lotação (cabeça/ha) & 1,64 & 2,45 & 2,30 & 1,27 & 1,42 \\
\hline Porcentagem de estabelecimentos que tem acesso a internet & $46 \%$ & $60 \%$ & $56 \%$ & $39 \%$ & $44 \%$ \\
\hline Porcentagem de estabelecimentos que tem trator & $61 \%$ & $71 \%$ & $77 \%$ & $40 \%$ & $51 \%$ \\
\hline Porcentagem de estabelecimentos que faz confinamento & $18 \%$ & $45 \%$ & $29 \%$ & $10 \%$ & $16 \%$ \\
\hline Porcentagem de estabelecimentos que usa ração, grãos e subprodutos agroindustriais & $66 \%$ & $100 \%$ & $79 \%$ & $57 \%$ & $63 \%$ \\
\hline Porcentagem de estabelecimentos que usa adubos químicos & $36 \%$ & $23 \%$ & $44 \%$ & $20 \%$ & $28 \%$ \\
\hline Porcentagem de estabelecimentos que usa corretivos do solo & $38 \%$ & $34 \%$ & $50 \%$ & $26 \%$ & $33 \%$ \\
\hline Porcentagem de estabelecimentos que recebe orientação técnica & $57 \%$ & $67 \%$ & $77 \%$ & $40 \%$ & $50 \%$ \\
\hline
\end{tabular}

Tratando das características socioeconômicas dos estabelecimentos agregados por município (Tabela 4), observa-se que o Cluster 2 concentra o maior percentual de estabelecimentos da agricultura familiar (LEI-11326 de 24-07-2017), com o valor de $51 \%$. Para o público objeto desta análise, a média de estabelecimentos classificados como de agricultura familiar é de $33 \%$ e os demais clusters se aproximam desse valor.

Tabela 4. Características socioeconômicas dos estabelecimentos produtores de bovinos de corte com mais de $\mathbf{5 0}$ cabeças no Bioma Mata Atlântica, agregados por municípios, segundo clusters e total Mata Atlântica

\begin{tabular}{|c|c|c|c|c|c|}
\hline Variável / Cluster & Cluster 1 & Cluster 2 & Cluster 3 & Cluster 4 & M. Atlântica \\
\hline Porcentagem de estabelecimentos familiares & $34 \%$ & $51 \%$ & $33 \%$ & $30 \%$ & $33 \%$ \\
\hline Porcentagem do valor da produção advinda dos bovinos em relação ao valor total da produção & $39 \%$ & $34 \%$ & $19 \%$ & $71 \%$ & $47 \%$ \\
\hline \begin{tabular}{|l|l|} 
Porcentagem da receita familiar que advém da agropecuária \\
\end{tabular} & $87 \%$ & $80 \%$ & $94 \%$ & $65 \%$ & $78 \%$ \\
\hline $\begin{array}{l}\text { Porcentagem de outras receitas (aposentadoria, trabalho fora, etc) em relação à receita total } \\
\text { do estabelecimento }\end{array}$ & $10 \%$ & $15 \%$ & $4 \%$ & $33 \%$ & $20 \%$ \\
\hline Porcentagem de estabelecimentos que tem energia elétrica & $93 \%$ & $95 \%$ & $94 \%$ & $90 \%$ & $91 \%$ \\
\hline
\end{tabular}


A porcentagem do valor da produção agropecuária que advém da produção de bovinos é maior no Cluster 4 , da ordem de $71 \%$, lembrando que esse é o cluster que mostrou menor grau de diversificação da produção. Já para os Clusters 2 e 3 (os mais diversificados) esse percentual cai para $34 \%$ e $19 \%$, respectivamente, enquanto no Cluster 1 é de 39\%. A maior parte da receita familiar provém da atividade agropecuária em todos os clusters, mas essa participação é menor no Cluster 4 - o de menor nível tecnológico e menor percentual de estabelecimentos familiares -, sendo aí da ordem de $65 \%$. No Cluster 4 é também onde se observa a maior participação de outras receitas (aposentadoria, trabalho fora, etc) na formação da receita total do estabelecimento, com uma média de 33\% (Tabela 4).

Por fim, a Tabela 4 nos aponta que a energia elétrica ainda não é uma realidade para $10 \%$ dos estabelecimentos do Cluster 4 . A média de estabelecimentos com acesso à energia elétrica entre os produtores de bovinos de corte com mais de 50 cabeças no bioma Mata Atlântica é de 91\%.

As Figuras 1 a 4 mostram a localização espacial dos quatro clusters da Mata Atlântica. Verifica-se que que o Cluster 2 e o Cluster 3, de maior nível tecnológico, concentram-se nos estados do Sul e Sudeste do país. O Cluster 1 (nível tecnológico intermediário) se distribui predominantemente desde o estado de Santa Catarina até o Sudeste da Bahia. Já o nível tecnológico baixo (Cluster 4) se distribui ao longo de toda a extensão do bioma, mas com baixa frequência nos estados de Santa Catarina e Rio Grande do Sul. A Figura 5 reúne a localização espacial dos quatro clusters. Análises adicionais com emprego de geoestatística são interessantes para explicar o efeito da localização espacial sobre a produção e produtividade dos diferentes agrupamentos.

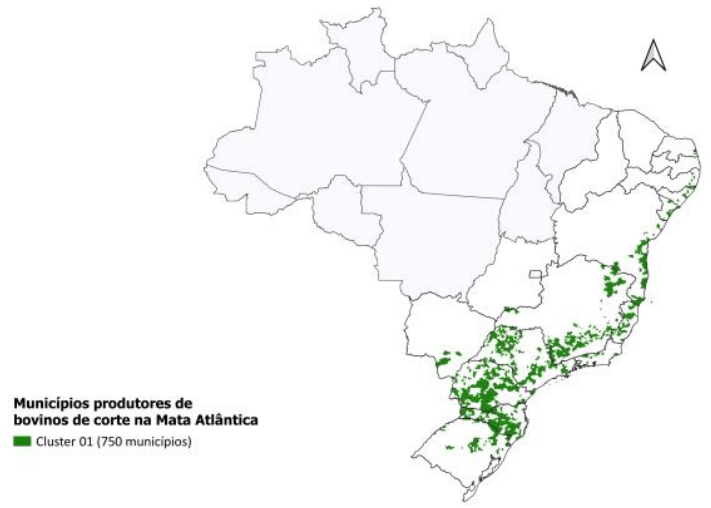

Figura 1. Localização espacial do Cluster 1

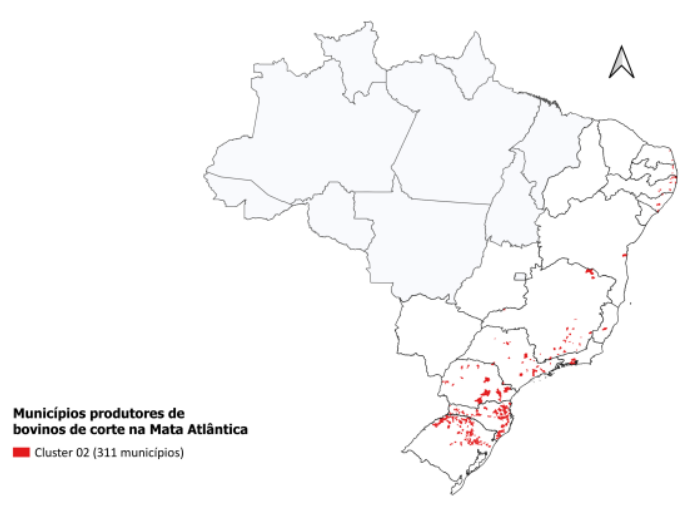

Figura 2. Localização espacial do Cluster 2 


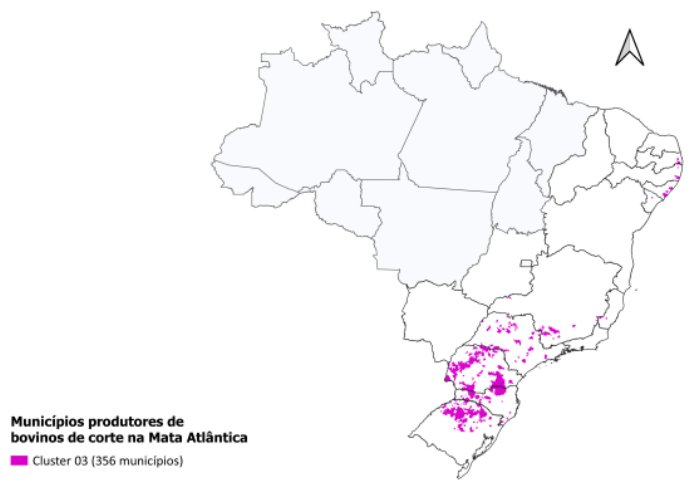

Figura 3. Localização espacial do Cluster 3

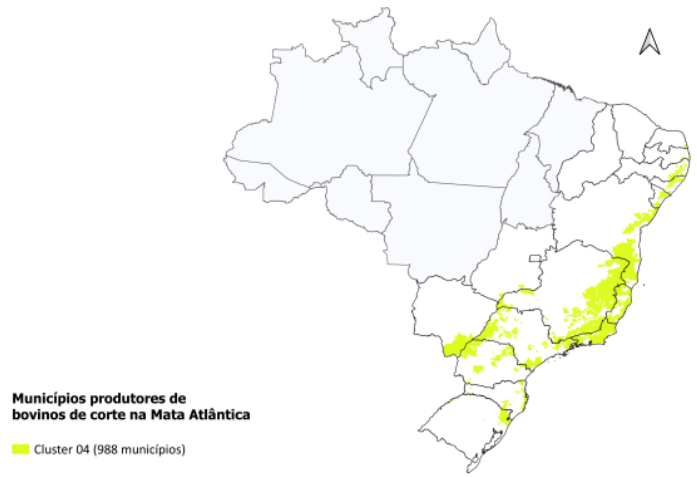

Figura 4. Localização espacial do Cluster 4

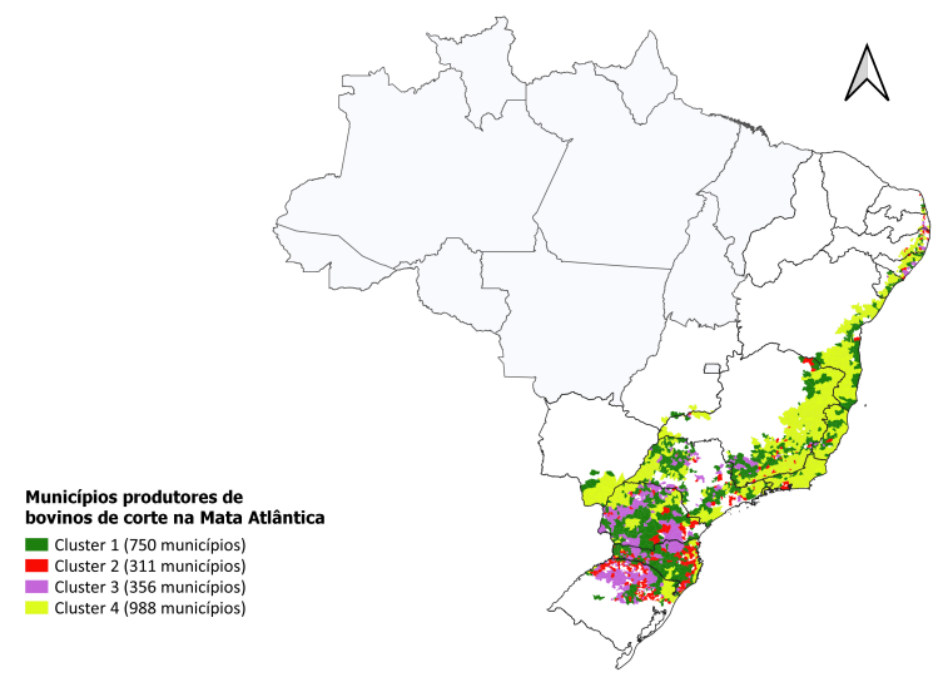

Figura 5. Localização espacial dos 4 clusters de municípios com produtores de bovinos de corte com mais de $\mathbf{5 0}$ cabeças no bioma Mata Atlântica.

\section{Conclusões}

Estudos como este são relevantes por mostrarem a realidade da produção praticada ao longo do país, podendo apontar as áreas prioritárias para ações de pesquisa, transferência de tecnologia e desenvolvimento, colaborando para a conservação dos recursos naturais do bioma.

Analisando os estabelecimentos agropecuários com mais de 50 cabeças de bovinos de corte do bioma Mata Atlântica, agrupados por municípios, pode-se diferenciar e caracterizar os sistemas de produção predominantes, permitindo elaborar o mapa da sua distribuição ao longo do território nacional, com enfoque principal no seu nível tecnológico e acesso a serviços como assistência técnica, internet e energia elétrica. Por esse mapa, podem ser melhor definidas áreas prioritárias para atuação institucional com a finalidade de melhorar a produção e a produtividade da pecuária regional.

Entre os estudos futuros, considera-se importante estabelecer comparações entre os dados dos dois últimos censos agropecuários (de 2006 e 2017) de modo a compreender a evolução dos sistemas de produção praticados no país nesse espaço de onze anos. 


\section{Referências}

Carvalho, T. B. de; Zen, S. de (2017). A cadeia de Pecuária de Corte no Brasil: evolução e tendências. Revista iPecege v.3, n.1, p. 85-99. DOI: 10.22167/r.ipecege.2017.1.85. Disponível em: <https://revista.ipecege.org.br/Revista/article/view/109>. Acesso em: 22 out. 2021.

Dempster, A. P., Laird, N. M., Rubin, D. B. (1977) Maximum likelihood from incomplete data via the EM algorithm. "Journal of the Royal Statistical Society". Series B (Methodological), Blackwell, v. 39, n. 1, p. 1-38.

Frank, E.; Hall, M. A.; Witten, I. H. (2016). The WEKA Workbench. Online Appendix for "Data Mining: Practical Machine Learning Tools and Techniques", Morgan Kaufmann, Fourth Edition.

DIAGNÓSTICO da pecuária de corte em Minas Gerais. Belo Horizonte: Sistema FAEMG, 2016. 147 p. Disponível em: $<$ http://www.faemg.org.br/Content/uploads/publicacoes/arquivos/Xcui1592321508129. pdf $>$. Acesso em: 22 out. 2021.

EMBRAPA. "Rede ILPF. BIOMA: MATA ATLÂNTICA". [S.1.] Disponível em: <https://www.embrapa.br/web/rede-ilpf/bioma/mata-atlantica>. Acesso em: 19 ago. 2021.

Fasiaben, M. do C. R., Oliveira, Almeida, Abreu. (2018) "Diferenciação e caracterização de municípios produtores de bovinos de corte no bioma Mata Atlântica". In: CONGRESSO DA SOCIEDADE BRASILEIRA DE ECONOMIA, ADMINISTRAÇÃO E SOCIOLOGIA RURAL, 56., 2018, Campinas. Transformações recentes na agropecuária brasileira: desafios em gestão, inovação, sustentabilidade e inclusão social: anais. Brasília, DF: SOBER, 2018. Não paginado.

Fundação SOS Mata Atlântica, INPE. (2021) "Atlas dos remanescentes florestais da Mata Atlântica, período 2019-2020”. Relatório Técnico. São Paulo. 73p.

Fundação SOS Mata Atlântica. (2021) "Desmatamento da Mata Atlântica cresce em dez estados". 2021. Disponível em: <https://www.sosma.org.br/noticias/desmatamento-damata-atlantica-cresce-em-dez-estados/> Acesso em: 19 ago. 2021.

IBGE. (2020) "Tabulação especial do Censo Agropecuário 2017 de estabelecimentos de bovinos de corte com mais de 50 cabeças agregados por município". Rio de Janeiro: Instituto Brasileiro de Geografia e Estatística, Coordenação de Agropecuária.

INCT-BioNat. "Mata Atlântica". [S.1.]. Disponível em: < bionat.iq.unesp.br/biomas/mata-atlantica/>. Acesso em: 19 ago. 2021.

Marcondelli, A. C. B., Nicodemo, M. L. F., Barioni Júnior. (2018) "Diversidade funcional em sistemas de produção pecuários no bioma Mata Atlântica". In: SIMPÓSIO BRASILEIRO DE AGROPECUÁRIA SUSTENTÁVEL, 9.; CONGRESSO INTERNACIONAL DE AGROPECUÁRIA SUSTENTÁVEL, 6., 2018, Viçosa, MG. Ciência, tecnologia e inovação para o desenvolvimento agropecuário sustentável: anais...Viçosa, MG: Biblioteca Central, Campus UFV, 2018. 142-146 SIMBRAS.

Pinto, L. F. G., Hirota, M., Rosa, M. (2021) "Atlas da Mata Atlântica e Mapbiomas se complementam no monitoramento do bioma". Disponível em: $<$ https://www.sosma.org.br/artigos/atlas-da-mata-atlantica-e-mapbiomas-se- 
complementam-no-monitoramento-do-bioma/> . Acesso em: 19 ago. 2021.

UNESCO. "World Heritage List". (2021) Disponível em: <http://whc.unesco.org/en/list/ $\geq$. Acesso em: 19 ago. 2021. 